\title{
Perspectiva de género(s) y feminismos en el campo de las Relaciones Internacionales. Trayectorias, identificaciones y perspectivas iberoamericanas
}

Perspective of gender(s) and feminisms in the field of International Relations. Ibero American trajectories, identifications and perspectives

Florencia Di Giorgio ${ }^{1}$

Leandro Sanchez $z^{2}$

Mariana Jacques ${ }^{3}$

\section{Resumen}

Las perspectivas críticas feministas en el campo de las Relaciones Internacionales aún permanecen en los márgenes de la disciplina, en pugna con los discursos y posiciones epistémicas ortodoxas del Norte Global, latitud que dio nacimiento a la disciplina y desde donde se exporta la mayor parte del corpus teórico.

Este trabajo se sitúa en los estudios críticos sobre dependencia académica para analizar 405 las formas en que las perspectivas de género(s) y los feminismos circulan en y desde los países de Iberoamérica en el ámbito de las Relaciones Internacionales.

Bajo esta premisa, en esta primera etapa nos propusimos describir cuantitativamente las particularidades de la circulación del conocimiento en el Sur y plantear si puede hablarse -o no- de una comunidad epistémica con reivindicaciones y conceptualizaciones propias. Para tal fin, abordamos los artículos con perspectiva de género(s) (447) publicados en las revistas iberoamericanas de Relaciones

\footnotetext{
${ }^{1}$ Maestranda en Estudios y Políticas de Género (UNTREF). Licenciada en Ciencia Política y Relaciones Internacionales (UCALP). Integrante del Centro de Estudios en Género(s) y Relaciones Internacionales (IRIUNLP) La Plata, Argentina. Correo electrónico: dgiorgioflorencia@gmail.com ID https://orcid.org/0000-00018163-1784

${ }^{2}$ Dr. en Ciencias Sociales (UNLP), Mg. en Metodología de la Investigación Social (Universita di Bologna), Lic. en Ciencia Política y Relaciones Internacionales (UCALP). Docente e investigador del Instituto de Investigaciones en Humanidades y Ciencias Sociales (IdIHCS-UNLP-CONICET), La Plata, Argentina. Correo electrónico: sanchez.leandro.e@gmail.com ID https://orcid.org/0000-0003-0931-0681

${ }^{3}$ Licenciada en Relaciones Internacionales (UNR). Integrante del Centro de Estudios en Género(s) y Relaciones Internacionales (IRI-UNLP), La Plata, Argentina. Correo electrónico: marianacristinajacques@gmail.com ID https://orcid.org/0000-0001-7641-4291
} 
Internacionales (236) de acceso abierto indexadas en la plataforma Latindex (13.809) durante los últimos diez años (2009-2019).

Palabras clave: comunidades epistémicas, feminismos y Relaciones Internacionales, circulación del conocimiento, perspectivas críticas.

\section{Abstract}

Critical feminist perspectives in the field of International Relations, still remain on the margins of the discipline, in conflict with the speeches and orthodox epistemic positions of the Global North where the discipline emerged and exports the majoritiy of the theoretical knowledge.

This work is situated in the critical studies on academic dependency to analyze the ways in which the perspectives of gender (s) and feminisms circulate in and from the Ibero-American countries into the field of International Relations.

Under this premise, in this first stage we set out to quantitatively describe the particularities of the circulation of knowledge in the South and to consider whether it is possible to speak -or not- of an epistemic community with its own claims and conceptualizations. In order to do that we address the articles with a gender perspective (447) published in the open access Ibero-American journals of 406 International Relations (236) indexed on the Latindex platform (13.809) during the last ten years (2009-2019).

Keywords: epistemic communities, feminisms and International Relations, knowledge circulations, critic perspectives.

\section{Perspectivas del quehacer científico}

En el desarrollo histórico de la Sociología de la Ciencia se pueden identificar dos grandes tradiciones que explican el quehacer científico: una de ellas entiende a la ciencia como institución específica y diferenciada que permite distinguir las actividades que sus miembros desarrollan; y otra que considera a la ciencia como una forma social de conocimiento que resulta, previa su certificación por dicha institución, en conocimiento verdadero (Fernández Esquinas y Torres Albero; 2009) ${ }^{4}$.

\footnotetext{
${ }^{4}$ La primera de estas perspectivas es representada por el aporte realizado por Robert Merton -considerado padre fundador de la Sociología de la Ciencia- y sus seguidores, y es entendida como la perspectiva "clásica"denominada también Escuela de Columbia-. La segunda de estas visiones es aquella conocida como Sociología del
} 
Este trabajo se ancla en la segunda tradición. Partimos fundamentalmente de la tesis de Knorr-Cetina, según la cual "los productos de la ciencia son construcciones contextualmente específicas que llevan las marcas de la contingencia situacional y de la estructura de intereses del proceso por el cual son generados" (Knorr-Cetina, 2005, p. 61). Fundamentalmente, el proceso de construcción de conocimiento tiene lugar como consecuencia de una cadena de decisiones y negociaciones.

A cada momento, lxs científicxs se confrontan con diferentes decisiones posibles y deben aplicar diferentes criterios para decidir. No obstante, la contextualidad observada en el laboratorio es permanentemente atravesada y sostenida por relaciones sociales que trascienden el sitio de investigación. Lxs científicxs interactúan hacia el interior, pero también hacia el exterior de su comunidad de especialidad. Es decir, no existe una separación entre fuerzas cognitivas y fuerzas sociales.

Respecto al comportamiento de aquellxs, Knorr-Cetina (1983) retoma las analogías económicas ${ }^{5}$ y las critica. Identifica a Bourdieu como el primero en entender la ciencia como una economía capitalista de mercado en donde lxs científicos luchan por el crédito entendido como capital simbólico y no como reconocimiento. Si bien adhiere al argumento de Bourdieu, considera sin embargo que el modelo sigue promoviendo una visión internista de la ciencia porque se sigue restringiendo la mirada a lxs propios científicxs y se lxs trata como aisladxs en un sistema auto- 407 contenido y cuasi-independiente.

La autora agrega a la metáfora económica dos cuestiones, pero nos detendremos en la segunda. Además de lxs científicxs que están en el laboratorio, Knorr-Cetina (2005) afirma que deben considerase a agentes del mundo extra-científico, tales como la rectoría de la universidad, personal administrativo de la institución, funcionarixs del organismo nacional de investigación, miembros o representantes de la industria, gerencia de una editorial, entre otros. En este sentido, supera la conceptualización de comunidades científicas entendidas como grupos de especialidad en un sistema autocontenido, para referirse a las mismas como "arenas transepistémicas de investigación", organizadas en términos de relaciones de recursos (Knorr-Cetina, 1996), de las cuales el/la investigador/a depende, y a las cuales recurre para que su investigación funcione.

La definición de dichas relaciones está permanentemente en juego, puede estabilizarse en algún momento mediante procesos de institucionalización $\mathrm{y}$

Conocimiento Científico, surge como respuesta crítica a la propuesta mertoniana y reúne un conjunto de autores diversos (Sarthou, 2013).

${ }^{5}$ Interés, capital, mercado, competencia, monopolio, oferta, demanda, etc. 
rutinización, pero lo que cuenta como recurso (ideas, instrumentos, materiales, líneas de acción) puede ser interpretado, ignorado o transformado, según cuál sea el juego.

Dentro de ese abordaje constructivista, como bien señala Hankinson Nelson (1993), hay una discusión sobre lxs agentes o sujetxs de la epistemología partiendo del supuesto de que la categoría no tiene contenido fijo o histórico, es decir, que no fue fijada de una vez por todas por la epistemología cartesiana (o cualquier otra).

Para las epistemologías feministas tales situaciones deben especificarse utilizando la categoría analítica género y diferencia sexual, categorías cuyo "contenido" y significado son dinámicos y multinivel y cuya relación con otras categorías y relaciones sociales (por ejemplo clase, cultura y raza), como así con el conocimiento, sigue siendo controvertido y central para las teorías.

Al situarse (y al mismo tiempo ser impugnados), lxs agentes de las epistemologías feministas difieren significativamente de los "individuos" abstractos (independientes del contexto y desencarnados) de epistemologías fundacionalistas. Un segundo supuesto que subyace en esa discusión es que los puntos de vista de lxs agentes de la epistemología no son aisladxs o aislables. Particularmente importantes en ese vínculo han sido los argumentos feministas que apuntan a la profunda inverosimilitud del "individualismo epistemológico": la visión del conocimiento como "un asunto individual... la actividad mental de los conocedores individuales que captan la única 408 verdad objetiva” (Addelson y Potter 1991: 12). Lxs feministas han argumentado que, para que los individuos tengan creencias y saberes, es necesaria la experiencia interpersonal (Bleier 1984; Code 1991; Jaggar 1983; Longino 1990; Nelson 1990; Scheman 1983).

Basándonos en los supuestos y desarrollos que se han esbozado previamente, proponemos el argumento de que los agentes generadores de conocimiento son comunidades y sub-comunidades, no individuos. Que las "comunidades epistemológicas” son agentes de la epistemología. Esta posición es compatible y está respaldada por la experiencia y el conocimiento feministas, lo cual indica que las comunidades son los lugares primarios -los principales generadores, repositorios, poseedores y adquirentes- de conocimiento.

La afirmación es que el conocimiento que hacemos como individuos es derivado. Más concretamente, sólo podemos saber lo que sabemos (o podríamos saber), para algún "nosotrxs". El "nosotrxs", según nuestro criterio, es un grupo o comunidad que construye y comparte conocimientos y estándares de evidencia; un grupo, en resumen, que es una "comunidad epistemológica". 
Ahora, la prioridad epistemológica de las comunidades no es una verdad evidente por sí misma (ni pretendida). Las comunidades epistemológicas son múltiples, históricamente contingentes y dinámicas: tienen fronteras difusas, evolucionan, se disuelven y se recombinan, y tienen una variedad de "propósitos" y proyectos.

El cambio que proponemos en términos de nuestra comprensión de los agentes de la epistemología significará que los estudios de cómo se genera el conocimiento comenzarán a partir de las historias, relaciones sociales y prácticas de las comunidades.

Lo anterior tiene implicaciones para el tipo de proyecto que emprendimos, indica que las discusiones al nivel de éste se consideran acertadamente como propuestas de investigación y que, en general, el hacer de la epistemología requiere más estudios empíricos, que a su vez tienen implicaciones de cómo entendemos y emprendemos la epistemología feminista ${ }^{6}$.

\section{Comunidades epistémicas

\section{Internacionales}

Uno de los debates más recientes en la teoría de las relaciones internacionales es la creciente crítica al "localismo" o "provincialismo" de una disciplina que, como señaló Stanley Hoffmann en 1977, ha sido mayormente una "ciencia social estadounidense". Este asunto ha sido retomado por autores como Holsti (1985), Wæver (1998), Smith (2000), Crawford, Jarvis y sus colaboradores (2001), para quienes las Relaciones Internacionales $(\mathrm{RI})$ continúan siendo una disciplina cuyos conceptos y modelos expresan intereses y visiones de Estados Unidos, mientras el Sur Global constituye la periferia.

Sin embargo, a partir, por un lado del cuestionamiento a la hegemonía anglosajona y su particular enfoque de lo que se considera relaciones internacionales y, por el otro, de la irrelevancia que el Sur Global tendría en su definición, se ha desarrollado un debate amplio, intenso y crítico de esa posición. Obviamente, analizar en detalle este debate excede los objetivos de este trabajo. Sin embargo, dos temas centrales del mismo merecen ser mencionados, pues ayudan a la compresión del planteo de la investigación que intentamos desarrollar.

\footnotetext{
${ }^{6}$ En relación con esto, los recientes argumentos posmodernos (y otros) han llamado la atención sobre los peligros de reemplazar los fundamentos "antiguos" por otros nuevos (aunque sin darse cuenta) al insistir en la inestabilidad y la naturaleza no fundacional de las categorías centrales de las epistemologías feministas, incluyendo, si no especialmente, el género (Flax 1987; Harding 1986, 1991; Hekman 1990).
} 
En primer lugar, nos posicionamos dentro de los enfoques críticos y su alejamiento de la ontología materialista e individualista propia del racionalismo, al tiempo que epistemológicamente intentamos practicar la "reflexividad teórica", es decir, la necesidad de reflexionar sobre el propio proceso de teorización, rechazando cualquier monismo metodológico, como también la pretendida neutralidad axiológica, que oculta su carácter ideológico sostenedor del status quo y esa función de "disciplinar la disciplina" al fijar los parámetros de lo que se debe estudiar y no.

En segundo lugar, se discute si lo producido en los países del Sur Global no es más que una serie de conceptos y definiciones "prestadas" de las teorías dominantes y usadas para resolver problemas específicos.

Se debate, entonces, si en el Sur solo se "mimetizan" los conceptos emanados en el centro (Acharya, 2013), o más bien se trata de un proceso de hibridación dinámico y contingente. Aclaramos que mimetizar (mimicrys) es un concepto desarrollado por las teorías poscoloniales, en particular Homi K. Bhabha, quien lo define como "el deseo de ser otro, reformado y reconocible, como sujeto de diferencia que es casi lo mismo, pero no exactamente" (Bhabha, 1994, p. 86). Sin embargo, en el debate reciente de las RI, mimetizar se usa en el sentido de emulación, imitación o simplemente de una importación acrítica de las teorías del centro.

Ahora bien, muchxs autorxs cuestionan que en el Sur Global se haya producido 410 una simple mimetización, sino más bien una incorporación "selectivamente apropiada y rearticulada dentro de contextos y discursos locales" (Smith, 2012). Por eso, más que una mimetización, se trataría de procesos de "hibridación", en los cuales se utilizan elementos del pensamiento de las corrientes dominantes de la disciplina, pero en el marco de un contexto y de forma que difieran de cómo se elaboraron en el centro.

En este contexto, el mundo no estaría entonces basado en una idea binaria de centro y periferia, sino que existiría lo que Bhabha denomina un "tercer espacio", un in

\footnotetext{
${ }^{7}$ La idea de hibridación fue desarrollada por Bhabha desde un enfoque psicoanalítico y literario, en gran medida derivado de su interés en el problema de cómo representar al "otro" en la literatura y en la academia (Ackermann, 2012, p. 12). Ese "otro" era el sujeto colonial, cuya experiencia de vida transcurría en la ambigüedad de estar ubicado (o haber estado) en un espacio colonizado por un poder imperial, que buscaba imponer su cultura como superior. Para Rita Abrahamsen, mediante el concepto de hibridación Bhabha se propuso demostrar justamente que los colonizados no fueron simplemente "víctimas pasivas, cuyas identidades fueron narradas en un proceso de una sola vía por las autoridades coloniales". En vez de ello, "la ambivalencia de las culturas y prácticas híbridas, la forma como ellas son 'casi lo mismo, pero no igual', es para Bhabha una señal de la condición de agente del colonizado y su capacidad de resistir a la dominación" (Abrahamsem, 2007, p. 117). La hibridación sería un símbolo de resistencia del colonizado, quien genera una contaminación de la ideología, la estética y la identidad imperial que ataca la dominación colonial (Kraidy, 2005, p. 58).
} 
between. Esta conceptualización implica un rechazo de la dicotomía universalismo versus particularismo. Las culturas no se consideran fijas o cerradas, sino que son abiertas e interconectadas, lo que establece los límites de una formación cultural "pura".

Lo cierto es que para nosotrxs, como señala Nederveen Pieterse, la hibridación se presenta de varias formas, lo que permite construir un continuum de hibridaciones. Puede existir un tipo de hibridación que se inclina hacia el centro, adopta el canon y mimetiza la hegemonía, pero también puede existir, en el otro extremo, una hibridación que diluye el canon, invierte lo actual y subvierte el centro.

Estos tópicos son cruciales en la compresión del principal interrogante de esta investigación: ¿podemos hablar de una comunidad epistémica con perspectivas de género y feminismos latinoamericanos en el campo de las Relaciones Internacionales?, ¿existe una identificación (para usar el concepto de Stuart Hall) de la que se pueda hablar?

El pensamiento es un punto de vista que estructura un modo de percibir y organizar la experiencia de la realidad. Pero, al mismo tiempo, los fenómenos del mundo de la realidad condicionan la estructuración del pensamiento. Pensamiento y realidad no son entidades discretas y separadas, ni es posible entre ellos establecer una prioridad ontológica; por el contrario, la organización del pensamiento y de la realidad 411 son procesos correlativos y mutuamente constitutivos. La complejidad reside en el carácter recursivo del proceso de constitución mutua.

Esto es sumamente significativo porque el mundo de la ciencia, como parte del mundo de la vida, se basa en una combinación de "sujeción" externa y "subjetivación" interna como diría Rabinow, es decir, las identificaciones (individuales y colectivas) se constituyen a sí mismos en términos de las explicaciones/interpretaciones a través de las cuales son identificados. Además, estas también tienen una función de legitimación.

\section{Diseño metodológico}

¿Cómo pasamos de estos supuestos a la práctica? Intentaremos, muy brevemente, exponer la estrategia de investigación para alcanzar los objetivos que desarrollamos.

\footnotetext{
${ }^{8}$ Obviamente tenemos en cuenta que la hibridación también ha sido criticada, no es que se desconozca ello. Por ejemplo, Amar Acheraïou (2011:7), cuestiona que la hibridación sea subversiva o contrahegemónica, sostiene que se trata, más bien, de un discurso que se adapta a lo que denomina las estructuras globales de poder neoliberal/neocolonial, lo que es resultado, en gran medida, de la ambivalencia e indeterminación de sus propuestas.
} 
A la hora de caracterizar la investigación existen tantas clasificaciones como autorxs, pero sintéticamente puede ser clasificada como una investigación explicativa, longitudinal, aproximada, retrospectiva y de tipo polietápico. Dentro de los enfoques mixtos planteamos el diseño transformativo secuencial. Y lo que vamos a presentar en este trabajo es la primera etapa, la descriptiva cuantitativa.

La estrategia de recolección de información utilizada sería, lo que a partir de la publicación de Webb et al. (1966), se conoce como investigación no reactiva. Esto es, la observación de archivos documentales, en este caso artículos de revistas científicas, que conforman nuestra unidad de observación.

En cuanto a las revistas, en la medida en que constituyen la materia prima de la base sobre la que descansó todo el proceso de investigación, resulta especialmente importante caracterizarlas, determinar qué tipo de información y de cuánta información se dispuso para realizar el trabajo, así como para qué se utilizó la información disponible.

Para ello, y este es un punto central ya que tomamos una decisión acorde a la posición epistemológica que sostenemos y tiene clara incidencia en todo lo que sigue, debemos hacer algunas aclaraciones.

En las últimas décadas, la obsesión por publicar en revistas indexadas en bases de datos de Web of Science (WoS-Clarivate) y Scopus (Elsevier) ha ido in crescendo en 412 todas las latitudes. Pertenecer al mainstream se ha convertido en sinónimo de calidad en una gran parte del mundo académico. Esta tendencia se ve cada vez más profundizada, incluso en regiones como América Latina, a medida que los sistemas de evaluación nacionales de la región dejan de valorar indicadores de calidad, relevancia y pertenencia.

Es evidente que los criterios de inclusión de Wos y Scopus son ambiguos y sesgados a sus propios objetivos. Aun así, la citación sigue siendo la medida aceptada por la comunidad científica como indicador de calidad y prestigio. Por eso, en este marco, se consideró que la adopción de la política de acceso abierto (AA) es una característica de alto valor al pensar la visibilidad de una revista, sus artículos, de una posición epistemológica, que era nuestro objetivo. ¿Por qué? Porque una revista es más accesible cuando no cobra por leer ni publicar, y no existe visibilidad real si no se acompaña de accesibilidad, y no hay accesibilidad más transparente que la del acceso.

De manera que, en los ecosistemas de comunicación científica, donde el valor del conocimiento como bien común es un elemento de desarrollo de la sociedad, se consideró aún más importante evaluar la visibilidad y accesibilidad con mayor énfasis 
en la misma sociedad, región en este caso, que en la ciencia mainstream ${ }^{9}$. Es por ese motivo que el relevamiento realizado se estructuró sobre la base de Latindex, proyecto pionero de la región, contrastado a su vez con SciELO y Redalyc.

Esta decisión implicó contar con una no menor cantidad de información y un acceso rápido a parte de la misma, el listado de revistas. De un total de 13.809 revistas iberoamericanas de ciencias sociales, 236 serían de Relaciones Internacionales.

A partir de aquí y una vez definido el "universo" que constituye la primera unidad de observación (las revistas), se procedió a la creación de una primera matriz de datos y la depuración de información.

Una vez caracterizadas las revistas, el paso siguiente fue identificar al interior de cada revista, en el período 2009-2019, aquellos artículos con perspectiva de géneros, nuestra unidad de análisis.

Ello implicó algunas cuestiones básicas respecto a las variables que debíamos considerar al momento de construir la segunda matriz: a) revisar las definiciones nominales con las que trabajamos, b) la codificación posterior y c) la operacionalización.

Cabe destacar que codificar los datos significa asignarles un valor numérico o símbolo que los represente. Es decir, a las categorías de cada ítem o variable se les asignan valores numéricos que tienen un significado. Esta "precodificación" fue 413 compleja y discutida, a sabiendas de que hay veces que un ítem no puede ser codificado a priori (precodificado), porque es muy difícil conocer cuáles serán sus categorías.

El resultado de ese proceso es un listado de 46 variables por las cuales fueron tamizados cada uno de los artículos identificados, para ser precisos 447 artículos.

\footnotetext{
${ }^{9}$ Una visibilidad integral necesariamente tiene que considerar los diversos tipos de uso que reciben las revistas. Si bien las citas son una de las medidas, y el modelo de acceso junto con la accesibilidad técnico-tecnológica son otras, es necesario considerar también el alcance que los artículos tienen en ámbitos no académicos, aspecto que no podemos esclarecer en esta etapa, pero que pretendemos analizar. De hecho, en la última década se ha impulsado el uso de métricas conocidas como Altmetrics -métricas alternativas- que permiten identificar la circulación de las producciones científicas en ámbitos no académicos. Por ejemplo, el DOI (Digital Object Identifier) en asociación con servicios de empresas tales como Altmetric.com o Plum Analytics ha incrementado el uso y la visualización de indicadores alternativos tanto en las webs de las revistas como en distintos servicios de información. Actualmente, para medir el alcance es factible utilizar los módulos que ofrece Open Journal System (OJS) para visualizar las descargas y vistas de cada artículo y con el plugin Paperbuzz que ayuda a visualizar métricas por artículo en relación con vistas y usos en Twitter y Wikipedia, entre otras fuentes.
} 


\section{Las revistas de Relaciones Internacionales}

Dentro del continuum que se extiende desde el internalismo hasta el externalismo respecto a las explicaciones sociológicas del quehacer científico, existen diferentes mixturas y uno de los aspectos centrales en la identificación de una comunidad epistémica es la circulación de saberes, la textualidad de las ciencias sociales, el diálogo entre lxs investigadores sobre la producción del conocimiento, su escritura y su exposición pública. Si bien esto acontece de diversas maneras, este trabajo toma como principal dispositivo para ello las revistas académicas.

En las Ciencias Sociales en su conjunto, la tradición en la exposición del conocimiento se ha construido a través de los libros. Éstos forman la secuencia textual que contiene las aportaciones más importantes y dan cuenta de los cambios en los paradigmas de los distintos campos de especialización. Sin que ello deje de tener un valor significativo, cada vez más, las revistas científicas constituyen el acervo que da forma al canon de cada una de las disciplinas de ese conjunto cognitivo que llamamos ciencias sociales.

Como bien se señaló en el apartado metodológico, el ecosistema de comunicación científica que se adoptó, por razones ya expuestas, fue de 234 revistas de Relaciones Internacionales de las 13.809 revistas iberoamericanas de ciencias sociales, por lo que el primer dato significativo es que este campo de estudio representa el 1,7\% dentro de las 414 ciencias sociales. Es decir, es sumamente pequeño.

Caracterizar esas 234 revistas es un paso necesario porque la díada autor-artículo está ligada a la formación del científico social y su proceso de socialización en un campo disciplinario, y ello tiene como condición de posibilidad lo que las revistas establecen.

Lo primero que hay que decir es que de acuerdo a su propia autodefinición temática el 61,1\% de esas revistas incluye a las Relaciones internacionales como un subtema entre otros, y sólo el $38,9 \%$ se consideran revistas de Relaciones Internacionales de manera excluyente.

Ese conjunto de revistas, en su mayoría, provienen de instituciones educativas $(63,7 \%)$ y de investigación $(20,5 \%)$ y son de naturaleza pública $(57,7 \%)$, lo que redunda en que la mayoría tenga un soporte de acceso abierto, en línea, (69,2\%) permitiendo una mayor circulación de los saberes allí expuestos.

Esto es muy interesante si se considera que las reglas institucionales de evaluación y edición han tenido como consecuencia que la producción de libros elaborados en un largo plazo sea cada vez más costosa en ingresos y visibilidad para lxs investigadxres. Y que, en la presentación de resultados de investigación, la tendencia actual es fraccionar 
el conocimiento y acotar en el artículo la producción científica, cuyo objetivo lo vuelve más eficiente en la respuesta a la presión de la evaluación institucionalizada del conocimiento cada vez más especializado ${ }^{10}$.

Las posibilidades del artículo académico de responder, dentro de un campo analítico, a la exigencia de las coyunturas sociales o políticas de una sociedad, le permiten volverse el referente textual en una comunidad académica que responde a la demanda de conocimiento, tanto por el mundo académico como por lxs tomadores de decisiones. La frecuencia de esa circulación, al menos en lo que a la publicación se refiere, muestra que semestralmente se renuevan los contenidos $(44,9 \%)$ y en menor medida cuatrimestralmente $(15,8 \%)$. El resto de las frecuencias se ubican muy por debajo.

Un dato muy significativo de y para el campo de las Relaciones Internacionales tiene que ver con el crecimiento exponencial de las revistas especializadas desde la década del 70 a la actualidad. Hay picos muy por encima de la tendencia que fue de 5,2 revistas por año desde las primeras 2, aún vigentes: 14 nuevas revistas en el 2013, 13 en el 2010, 12 en el 2014 y en el 2000. Este dato cobra mayor relevancia cuando se lo coteja con la nacionalidad de origen ya que el incremento se corresponde con la emergencia de un gran número de revistas en Brasil. De hecho, de las 71 revistas que aporta Brasil, el 80\% se crearon a partir de la década del 2000.

En magnitud, después de Brasil siguen México con 35, Argentina con 34, España con 27, 11 Ecuador, Colombia con 10 y Chile con 9.

Las revistas son unidades expositivas que poseen cualidades variables según el campo analítico que desarrollan, pero, sobre todo, según el periodo histórico de la disciplina en el que se producen los textos. En esta modalidad escritural, la innovación o la continuidad dentro de un campo de especialización aparece explícita frente a la tradición establecida del conocimiento en la cual el texto se ubica. Por lo tanto, teniendo en cuenta que las Relaciones Internacionales son una disciplina eminentemente anglosajona pero que el conocimiento es situado, la lengua en que se escribe es una cualidad no menor. Estas revistas plantean la posibilidad de escribir en diversas lenguas. El 39,3\% sólo en castellano, el $15 \%$ sólo en portugués y el 0,9 en inglés. El 23,1\% promueven artículos en esas 3 lenguas y sólo el 0,9 en otros dialectos.

\footnotetext{
${ }^{10}$ La exigencia de rapidez en la presentación de los resultados de investigación ha traído como consecuencia una creciente presión en contra de la producción del libro, como modalidad de exposición unitaria e integral de los resultados de un prolongado proceso de investigación.
} 


\section{Los artículos con perspectiva de géneros:} caracterización general

Si se baja un nivel empírico de observación para concentrarse en los artículos con perspectiva de género existentes en estas revistas, habría que subrayar que representan un 1,7\% (447 artículos). Ese 1,7\% implica una media de 44 artículos por año, sin embargo la tendencia es creciente, en 2017 se publicaron 70, en 201879 y en 201972. Esto es, el $49 \%$ de los artículos con perspectiva de géneros se publicaron en los últimos 3 años del lapso abarcado.

Ese incremento se corresponde, en parte, al también creciente número de revistas. De hecho, el 39,7\% de los artículos fueron publicados dentro de los primeros 10 números de las revistas y si se suman los publicados en la siguiente decena llegan al $54.5 \%$.

Los criterios de cientificidad que define la línea editorial de cada una de las revistas académicas -explícitos o implícitos- cubren desde los requisitos que se consideran indispensables y miden la objetividad del conocimiento, hasta la construcción de un determinado tipo de evidencia empírica con la que se valida lo científico o no de un texto, pasando por la filiación teórica y la consistencia metodológica.

Un aspecto relevante en ese sentido es que, de acuerdo a las normas de 416 publicación de cada revista, la inmensa mayoría son definidos como artículos $(76,4 \%)$ y en menor medida como ensayos $(7,1 \%)$ y reseñas $(6,9 \%)$. Esto es importante porque en la definición del formato artículo subyace cierta definición de objetividad y está presente en el conjunto de criterios elaborados por los comités editoriales de las revistas académicas, criterios que se convierten en las condiciones necesarias para la valoración de los artículos propuestos por los autores y a partir de los cuales los textos tienen o no posibilidades de ser publicados en una determinada revista.

Las políticas académicas vigentes han estimulado la producción de artículos en revistas arbitradas con normas estrictas en su formato, reglas editoriales que empiezan por los límites en la extensión en que debe ser presentado el original para ser considerado para su publicación. Ello porque la tendencia actual es fraccionar el conocimiento y acotar en el artículo la producción científica, cuyo objetivo lo vuelve más eficiente en la respuesta a la presión de la evaluación institucionalizada del conocimiento cada vez más especializado. De ahí que la mayoría de los artículos no superan las 20 carillas. 
No sólo los artículos con perspectiva de géneros son acotados en su formato, sino que su ubicación al interior de un número específico de cada revista no los ubica en el centro de la escena (a excepción de los dossiers sobre la temática). Las revistas relevadas tienen un promedio de 300 páginas por número o volumen y sólo 79 aparecen en las primeras 40 páginas.

Las posibilidades del artículo académico de responder, dentro de un campo analítico, a la exigencia de las coyunturas sociales o políticas de una sociedad, le permiten volverse el referente textual en una comunidad académica que responde a la demanda del conocimiento sociológico, tanto por el mundo académico como por los tomadores de decisiones.

Siguiendo la tendencia que las propias revistas marcan los artículos con perspectiva de géneros también se escriben mayoritariamente en español $(61,4 \%)$ y portugués $(27,8 \%)$.

\section{Hacia una nueva cartografía de saberes}

Una idea central en la epistemología feminista es que "el conocimiento está/es siempre socialmente situado" (Harding, 2004, p. 7). Los productos de la ciencia son construcciones contextualmente específicas que llevan la marca de las experiencias, circunstancias y perspectivas físicas, psíquicas y sociales particulares de los sujetos; es 417 decir no sólo su corporalidad, sus valores, sus habilidades, sus estilos cognitivos, sus relaciones epistémicas con otros sujetos cognoscentes, etc., sino también su localización social: género, orientación sexual, raza, etnicidad, edad, estatus familiar, roles y relaciones sociales, su identidad social atribuida y su identidad subjetiva (del Moral Espín, 2012).

Con el propósito de contribuir a la construcción de nuevas cartografías de saberes, en nuestro trabajo examinamos el corpus de publicaciones por identidad de género, etnia, rango etario, nacionalidad, adscripción institucional, situación académica y disciplina principal de lxs autorxs. Asimismo, identificamos si los trabajos fueron escritos de forma individual o de manera colaborativa.

Lo que intentamos hacer al tamizar los artículos con las variables indicadas anteriormente fue abrir la "caja negra" de lo que podría(n) denominarse comunidad (es) epistémica(s) feministas $\mathrm{y} / \mathrm{o}$ con perspectiva de géneros en las Relaciones Internacionales, para poder dilucidar su existencia (o no) y a su vez acercarnos al proceso de producción y circulación de conocimientos. 
En el período 2009-2019 identificamos la participación de 588 autorxs en la producción de los 447 artículos con perspectiva de géneros publicados en las 243 revistas indexadas de Relaciones Internacionales en Iberoamérica. Vale aclarar que la descripción realizada en este apartado está basada en los datos obtenidos para Autor 1 $(\mathrm{A} 1)^{11}$, lo cual representa el $73 \%$ del universo de autorías.

Si bien partimos del sentido controversial de las variables identidad de género ${ }^{12} \mathrm{y}$ etnia ${ }^{13}$ respecto a su contenido, significado y representatividad, su inclusión en la investigación tuvo por fin detectar aquellos casos en donde apareciera una autoidentificación explícita, relacionada a un pronunciamiento político de resistencia, de denuncia o bien, como una forma de activismo cultural e intelectual de identidades diversas.

En nuestro relevamiento pudimos observar que los casos autoidentificados como masculinidad trans, femeneidad trans, queer $u$ otros, apenas superan el 2,0\%. Esto demuestra una exclusión de las personas trans como sujetxs de conocimiento con posibilidades de adquirir los capitales culturales que se producen en la academia (Bello Ramírez, 2018). Algo similar ocurre con la variable etnia, con tan sólo 16 autorxs afrodescendientes y 1 proveniente de pueblos originarios.

Al cruzar ambas categorías observamos, por defecto ${ }^{14}$, que el $74 \%$ de autorías corresponden a mujeres cisgénero de origen caucásico (seguido por un 16,5\% de 418

\footnotetext{
${ }^{11}$ Los artículos relevados fueron tamizados por 46 variables en la segunda matriz de datos. Para las variables identidad de género, nacionalidad, rango etario, adscripción institucional, etnia, situación académica y disciplina principal, realizamos una apertura en Autor 1 (A1), Autor 2 (A2), Autor 3 (A3) y Autor 4 (A4) con el objetivo de recopilar información de los trabajos individuales y de aquellos realizados de forma colectiva (de 2 a 4 autores).

12 El concepto de identidad de género es una noción teórica compleja, que alude tanto a las atribuciones que las figuras significativas otorgan al/la niño/a como también a las representaciones que brinda el orden cultural imperante acerca de los modos de la(s) femineidad(es) y de la(s) masculinidad(es) Y, al mismo tiempo, es una conceptualización que representa la vivencia íntima que tiene el individuo sobre sí mismo (Gamba, 2017)

${ }^{13}$ En un nivel abstracto, tanto la raza como la etnicidad contienen un discurso sobre los orígenes y sobre la transmisión de características a través de las generaciones. Las identificaciones raciales utilizan aspectos fenotípicos como una clave para la categorización, pero se cree que se transmiten dentro de las generaciones (a través de la 'sangre'); de ahí que sea importante el origen ancestral; de igual manera, la etnicidad trata del origen en una geografía en la cual una persona absorbe la cultura de un lugar (casi 'en la sangre') de las generaciones anteriores. Más prácticamente, si bien la etnicidad se refiere a la localización en una geografía cultural, pudiera darse el caso de que los rasgos fenotípicos que utiliza el discurso racial se distribuyan a lo largo de esa geografía. Asimismo, se pueden construir las identificaciones raciales dentro de una categoría racial, y viceversa, de manera que cualquier persona puede tener tanto una identidad racial como una étnica (Wade, 2000).

${ }^{14}$ El muestreo de las variables identidad de género y etnia la realizamos en base a la autoidentificación explícita por parte de la, el o le autor, ya sea en el propio artículo relevado para los fines de la investigación, en otros artículos o en sus redes sociales.
} 
varones cis del mismo origen), reproduciéndose en el campo de las RI, al igual que en otras disciplinas de las ciencias sociales, un predomino de sujetxs de conocimiento occidentales, blancos/as y heterosexuales ${ }^{15}$. Esta situación influye directamente en el debate sobre quiénes son sujetxs y quienes son objetos de conocimiento en los estudios de géneros y feminismos en las RI que circulan en la academia.

Respecto a la edad de lxs autorxs, sobresale la producción académica de personas con más de 40 años (24\%), seguida por el grupo etario de 33 a 40 años (18\%). Sin embargo, en los últimos 3 años del período abarcado, se produjo un importante ascenso de la participación de las generaciones más jóvenes ${ }^{16}$. Esto podría interpretarse como el correlato del protagonismo que asumieron los activismos feministas y disidentes jóvenes en la región en el último lustro ${ }^{17}$.

Siguiendo con la descripción de las variables, cuando se coteja el rango etario con la situación académica de lxs autorxs al momento de realizar la publicación, se observa claramente que la edad va acompañada de una mayor formación profesional, con titulaciones de magisters (28\%) y doctorados (47\%).

Este dato puede estar vinculado, entre otras cuestiones, a una mayor profusión de los estudios de géneros y feminismos en los programas de posgrado, siendo menos frecuente su incorporación en las mallas curriculares de las carreras de grado. Como señalan Rovetto, Camusso, Caudana y Figueroa (2017), la posibilidad que tienen de 419 emerger aquellos problemas de investigación históricamente no vistos supone, como requisito previo, la configuración de posibilidades epistemológicas que abran espacios dentro de los cuáles éstos puedan ser pensados ${ }^{18}$.

\footnotetext{
${ }^{15}$ Ver Alarcón, 1990; Alexander y Mohanty, 1997; Mohanty, 1991; Trinh, 1978.

${ }^{16}$ De 4 documentos de trabajo publicados en 2016 en el rango etario 26-32 años, se produjo un salto a 20 artículos en el 2017, manteniéndose en 13 artículos los próximos dos años.

${ }^{17}$ Según Larrone y Ponce (2019), cuando se habla de la implosión de los feminismos en la región en los últimos años, se hace referencia fuertemente a lo generacional como fuerza impulsora de este fenómeno. La autora, a su vez, identifica al movimiento \#NiUnaMenos en Argentina -dirigido en contra de los femicidios y la violencia hacia las mujeres que luego se replicó en distintos países de la región- como una de las principales experiencias que permitió multiplicar la voz de las mujeres y disidencias, no sólo en las calles, sino también en la academia, sindicatos, partidos políticos, etc.

${ }^{18}$ Los aportes que se han preocupado por cuestionar los saberes hegemónicos en las ciencias sociales e incluso interpelar los modos de producirlos, son los menos conocidos y difundidos en las instituciones de educación superior (Fox Keller, 1985; Fox Keller y Longino, 1996; Haraway, 1991; Harding, 1986; Maffía, 2005; Maffía, 2007). Estos avances teóricos y sus consecuentes producciones de evidencias empíricas permanecen invisibilizadas en los contenidos formativos de la mayoría de las disciplinas científicas (Rovetto, et al, 2017, p. 134).
} 
El tipo de documento también se encuentra vinculado con las variables nombradas anteriormente. A mayor edad, mayor formación académica y, a su vez, mayor cantidad de publicaciones de artículos originales ${ }^{19}$. Ahora bien, la frecuencia de las publicaciones por autxr en las revistas indexadas es muy baja. Este dato podría indicar que los temas abordados en los artículos resultan más bien de investigaciones esporádicas antes que de proyectos de largo plazo, sin embargo, como planteamos en el primer apartado de este trabajo, no solo se deben tomar en cuenta las motivaciones personales, sino también el entorno social e institucional que orienta y reorienta las motivaciones de lxs científicxs, y de las mismas instituciones que los acojen (Sarthou, 2013).

Otro aspecto relevante tiene que ver con que el $67 \%$ de los artículos son de única autoría, lo cual indica que, en el período estudiado, no abundaron los trabajos colectivos. Si se contrasta esta situación con la filosofía y praxis feministas -las cuales sientan sus bases en la colaboración, la horizontalidad, la diversidad- podríamos decir que dicha praxis aún se encuentra en proceso de construcción en la academia, y que debe abrirse paso (no sin resistencias) en el marco de sistemas de producción y evaluación basados en la competencia, donde la práctica generalizada de evaluación resulta ser la valoración del rendimiento de investigadorxs a título personal, antes que el resultado de la labor conjunta de equipos de investigación.

Ahora bien, en las publicaciones realizadas entre 2 o más autorxs, se puede observar un vínculo colaborativo entre investigadorxs más jóvenes, con estudios de grado y aquellxs de mayor edad y nivel de formación académica. Resulta común que en los trabajos donde participan varias personas, las que tienen más experiencia y acervo académico oficien de mentorxs y/o tutorxs durante el proceso de producción académica.

Contemplando la nacionalidad de lxs autores, se puede observar cierta correlación con la cantidad de revistas indexadas de Relaciones Internacionales desagregadas por nacionalidad, lo cual se desarrolló en el apartado anterior. Brasil es el país iberoamericano con mayor número de interesadxs (146) en estudiar las RI con perspectiva de géneros, seguido por Argentina (58), España (53) y México (46).

Al cotejar la nacionalidad con la adscripción institucional, se observa un dato significativo respecto a la circulación territorial del conocimiento. La comunidad científica en el caso brasileño se encuentra distribuida en universidades e instituciones

\footnotetext{
${ }^{19}$ Mientras que lxs autores con estudios de grado publicaron en el tiempo analizado 67 documentos de este tipo, aquellxs con formación de posgrado publicaron 227.
} 
federales, estaduales y privadas de distintos estados provinciales ${ }^{20}$, por lo cual inferimos que existe una distribución mucho más federal respecto a los programas de estudio e investigación con perspectiva de géneros. Sin embargo, en Argentina, España y México, la mayor parte de lxs autorxs adscriben institucionalmente a las universidades nacionales radicadas en la capital de dichos países ${ }^{21}$, evidenciándose una mayor centralización de los programas de investigación y de la producción académica en las grandes urbes. Whitley (2012) señala que la concentración del control de los recursos desalienta la búsqueda de metas de investigación diferentes o el desarrollo de nuevos enfoques; asimismo, bajas tasas de movilidad entre organizaciones también restringen la innovación intelectual, al reducir la velocidad y la frecuencia de circulación de nuevas ideas y hacen más difícil el ingreso de ideas externas al instituto o campo.

Respecto al encuadre disciplinario de lxs autorxs que publicaron artículos académicos, existe cierta diversidad. Este dato es importante ya que cada disciplina tiene sus propias categorías para comprender viejos y nuevos problemas, a su vez, cada una corrige a su manera visiones sesgadas, incorpora datos, realiza nuevas preguntas o propone nuevos modos de conocer.

La mayoría de lxs autorxs son formadxs en Relaciones Internacionales (24\%). A esta disciplina le siguen el Derecho (15\%), la Ciencia Política (11\%) y la Antropología (5\%). Ahora bien, la cantidad de autorxs según el campo disciplinario al cual pertenece, 421 no se distribuye de la misma manera en los distintos países. Tomando los casos más significativos, observamos que en Brasil prevalecen lxs internacionalistas, posicionándose en segundo lugar lxs profesionales del Derecho; mientras que en Argentina, son lxs politólogxs quienes llevan la delantera, secundados por lxs internacionalistas. En México, si bien la mayoría de lxs autorxs son internacionalistas, lxs antropólogxs ocupan un lugar destacado en la producción académica.

\footnotetext{
${ }^{20}$ Universidade Federal da Bahía, Pontifícia Universidade Católica do Rio de Janeiro, Universidad Estatal de Campinas, Universidade de São Paulo, Universidade Federal da Paraíba, Universidade Federal de Pernambuco, Universidade Federal de Santa Catarina, Universidade Federal do Paraná, Universidade Federal Fluminense, Pontifícia Universidade Católica de São Paulo (PUC-SP).

21 Universidad de Buenos Aires, Universidad Autónoma de Madrid, Universidad Complutense de Madrid y Universidad Nacional Autónoma de México.
} 


\section{Análisis de los discursos teórico/conceptuales ideológico/políticos}

En el proceso de identificación de una (o varias) comunidad(es) epistémicas(s) feministas y/o con perspectiva de géneros en las Relaciones Internacionales, debemos detenernos, a su vez, en el análisis de los discursos teórico/conceptuales, como así también, ideológico/políticos que dentro de la misma circulan.

Si bien excede a este trabajo la realización de una arqueología de los discursos planteados en los artículos relevados, tener un panorama de la distribución epistemológica y política de las investigaciones con perspectiva de género en RI es una instancia necesaria para la comprensión integral de la comunidad cuya existencia pretendemos dilucidar.

Para la distribución epistemológica nos basamos principalmente en la tipología de Sandra Harding, quien distingue entre a) feminismo empiricista, b) feminismo "de punto de vista" (standpoint feminism) y c) feminismo postmoderno (Harding, 1996 ${ }^{22}$. En relación al criterio político (asunciones político-filosóficas subyacentes) partimos de la tipología elaborada por Alison Jaggar, quien distingue entre tres variedades de teorización: a) el feminismo liberal, b) el feminismo socialista/marxista y c) el feminismo radical (Salomón, 2002:33), a la cual adherimos, la corriente de feminismos críticos y el posmodernismo feminista ${ }^{23}$.

\footnotetext{
${ }^{22}$ Feminismo empiricista: consideran que el sexismo y el androcentrismo presentes en la investigación científica son sesgos sociales que es posible corregir mediante la estricta adhesión al método científico. Presupone la posibilidad de conocer la realidad. Feminismo del punto de vista: sostienen que la posición subyugada de la mujer les abre la posibilidad de un conocimiento más completo. El punto de vista de las mujeres, por lo tanto, permite desarrollar un "punto de vista" moral y científicamente preferible para las interpretaciones y explicaciones de la naturaleza y la vida social. Feminismo posmoderno: rechazan la idea de que pueda existir un "punto de vista femenino". No existe ninguna "experiencia femenina" ni punto de vista sobre el cuál sea posible construir una teoría del mundo social y político. Les interesa mostrar cómo los discursos y las estructuras dominantes y hegemónicas están profundamente imbuidas por la ideología patriarcal y el dominio masculino.

${ }^{23}$ Feminismo liberal: defiende los valores de libertad, dignidad, igualdad y autonomía y a partir de los mismos denuncia la injusta discriminación de la mujer. Sus propuestas políticas buscan revertir esa situación y alcanzar la igualdad de derechos con los hombres en las distintas esferas de la actividad humana. Feminismo marxista: aparece, en parte, como una crítica a la teoría feminista liberal. La opresión de las mujeres no es resultado de las actuaciones intencionadas de individuos sino un producto de las estructuras políticas, sociales y económicas asociadas con el capitalismo, en particular con el sistema de clases. La desigualdad socioeconómica está estrechamente vinculada a la división sexual y desigual del trabajo. La liberación de la mujer se concibe como parte de una lucha más amplia contra el sistema de opresión capitalista. Feminismo radical: se centra en la crítica al patriarcado, el sistema que hace posible el dominio de la mujer por parte del hombre. La opresión de las mujeres no puede erradicarse únicamente reformando las leyes o compartiendo responsabilidades (liberales) ni compartiendo en pie de igualdad las instituciones políticas y económicas (como las marxistas) sino mediante una
} 
Los discursos son una forma de acción, por eso, identificar que, de la totalidad de 453 artículos, el $68,2 \%$ se inscribe en términos epistemológicos dentro del punto de vista feminista nos permite interpelar la supuesta distribución que considera al postmodernismo feminista a la par del primero en la disciplina de las RI. Mónica Salomón (2002) ha afirmado que "en Relaciones Internacionales los enfoques feministas hicieron su aparición a fines de los años ochenta y de la mano de la entrada de los postmodernismos en la disciplina. Estos enfoques se autodefinen bien como pertenecientes a la postura epistemológica del feminismo de punto de vista, bien como postmodernos" (p.35); no obstante, en el lustro analizado en la región iberoamericana, la epistemología posmoderna $(17,4 \%)$ está muy alejada de las producciones de punto de vista, y más aún aquellas elaboradas desde el enfoque epistemológico del feminismo empiricista (11\%).

La epistemología del punto de vista feminista pretende no sólo de atender las experiencias femeninas, sino también otorgar un valor epistemológico privilegiado a un sujetx conocedorx determinadx, a aquél que tiene un punto de vista feminista. En este sentido, podríamos conjeturar, a priori, que el enorme desnivel entre las inscripciones epistemológicas de los artículos puede deberse al intento de saldar la ausencia de casi un siglo de las mujeres y disidencias sexo genéricas en la historia y en los procesos de toma de decisiones en las cuestiones internacionales, o bien por la 423 escasa permeabilidad de la disciplina a construcciones teóricas vinculadas a la deconstrucción de las estructuras.

Ahora bien, esta identificación de unx sujetx epistémico y enunciativo privilegiadx puede construirse de variadas formas, por eso cruzar este análisis con las posiciones políticas desde las cuales se plantea tal sujetx, nos permite ampliar nuestro marco de comprensión. Si, a muy grandes rasgos, el objetivo macro del feminismo es propugnar por "un cambio en las relaciones sociales que conduzca a la liberación de la mujer -y también del varón ${ }^{24}$ a través de eliminar las jerarquías y desigualdades entre los sexos" (Gamba, 2007, p. 142) las formas en que tal objetivo ha sido planteado son muy diversas.

Ya sea desde los feminismos liberales, radicales y marxistas durante la segunda ola del feminismo, hasta los posicionamientos más críticos y deconstructivistas de la

\footnotetext{
"reconstrucción radical de la sexualidad". Identifica la situación de opresión y asimetría de los géneros como una cuestión estructural. Feminismos críticos: rechazan la idea de un grupo unificado y plantean la pluralidad de sujetxs. Ponen el énfasis en sujetxs situados, contextualizados. Posmodernismo feminista o decostruccionismo: se alejan de las definiciones y del sujetx presituado, al que proponen como agotado.

${ }^{24}$ Vaya la salvedad de la falta de interseccionalidad y reconocimiento de las diversidades.
} 
identidad y el sujeto durante los '80 -'90 el sentido y las modalidades de construcción del movimiento y su teoría ha mutado a lo largo del tiempo. Por eso, de los 309 artículos que construyen un punto de vista feminista, sus adscripciones ideológicaspolíticas se distribuyen de forma heterogénea, sobre todo, entre feminismo radical $(33,98 \%)$, liberal $(29,1 \%)$ y críticos $(28,8 \%)$ de manera que los discursos varían completamente.

En tanto el primero tiene una cosmovisión estructural de la opresión que viven las mujeres y el sexismo como dispositivo de dominación propio del patriarcado; las segundas se manejan en posiciones más superficiales y no contestatarias al ordenamiento sistémico. Por su parte, los posicionamientos críticos, rompen con el lenguaje de la univocidad planteado durante la segunda ola, disputan el pretendido "sujeto feminista" homogéneo y plantean la necesidad de atender a las diversas experiencias y las opresiones dentro de los feminismos que distan de ser uniformes; no obstante, esta disolución de un falso "nosotras" no implica una ruptura de las redes de solidaridad, sino que es un llamado a romper con la idealización de un solo punto de vista.

Por eso, los artículos inscriptos en corrientes políticas críticas oscilan entre el punto de vista $(65 \%)$ y el posmodernismo $(32,9 \%)$ como epistemologías, en tanto por coherencia interna resultaría incongruente que abordaran fenómenos y/o construyeran 424 sus signos discursivos en el marco del empirismo cuyos principios y fundamentos siguen la línea positivista, afirmando que no son los métodos los que están "mal" y que puede hacerse "buena ciencia" eliminando los sesgos sexistas de quien aplica tales métodos sin, por lo tanto, cuestionar los mecanismos de producción de conocimiento más tradicionales. Por eso, al contrario, el feminismo liberal desarrolla buena parte de sus artículos (30\%) en el marco del empirismo feminista.

Y por esta coherencia teórico-político-discursiva es que el 96\% de los artículos posmodernos oscilan entre las corrientes políticas críticas (ya explicadas) y posmodernas/deconstructivistas que quiebra completamente con el concepto de identidad y las pretensiones de verdad. Si entendemos que epistemológicamente lxs feministxs posmodernxs "comparten un profundo escepticismo respecto a los enunciados universales (o universalizadores) sobre la existencia, la naturaleza y las fuerzas de la razón, el progreso, la ciencia, el lenguaje y el 'sujeto/yo" (Flax, 1986, p. 26) y lo "único" y "verdadero" son mitos o metanarrativas que se han utilizado para oscurecer y reprimir las diferencias, entonces, este enfoque exige utilizar fundamentos que permitan investigar las identificaciones fragmentadas que crea la vida moderna. 
Por lo tanto, difícilmente pueda integrarse esto con posiciones que no problematicen la univocidad delx sujetx.

\section{Una lupa más allá de las corrientes políticas}

Ahora bien, entendiendo que acotarnos a sólo cinco corrientes dentro de la tipología política restringiría las posibilidades de análisis, es que elaboramos una variable adicional desagregando la misma, la cual incluye los siguientes registros: feminismo institucionalista (114 artículos); feminismo de la igualdad (65 artículos); feminismos decoloniales (39 artículos); teoría queer (25); feminismo de la diferencia (13 artículos); feminismos indígenas (12 artículos); masculinidades (11 artículos); ecofeminismo (9 artículos); feminismos populares (8 artículos); feminismos negros (6 artículos); transfeminismos (5 artículos); lesbofeminismos (1 artículo).

Con el reparo de que este proceso de clasificación se encuentra condicionado por nuestra situacionalidad a la hora de leer los artículos, como así también por los parámetros elegidos para codificar cada variable, llevamos adelante el proceso de clasificación bajo el principio de que las variables codificadas son el resultado de una construcción analítica coyuntural dentro del campo de los estudios de género y feministas que no pretenden representar exhaustivamente todo el universo teóricodiscursivo de esta área.

Es decir, las variables propuestas fueron una aproximación más bien organizadora a fines prácticos, no exhaustivas, y no pretendió ser una propuesta de estructuración unívoca y universal en donde cada variable sea excluyente. Al contrario, creemos y sostenemos que las variables confeccionadas más bien se relacionan y definen mutuamente.

Hecha esta aclaración lo primero que podemos observar es que, si descartamos aquellos artículos clasificados bajo el registro de "otros" (82) y "no se identifica" (63) que abarcan el 32\% del total, del $68 \%$ restante, $39,5 \%$ se concentran en el feminismo institucionalista y de la igualdad, a los cuales, si sumamos los números del feminismo de la diferencia $(2,9 \%)$ nos da un total de $42,4 \%$ de los artículos concentrados en tendencias políticas vinculadas a discursos y conceptos, sobre todo, del feminismo liberal y, en menor medida, radical $^{25}$.

\footnotetext{
${ }^{25}$ Sólo tres artículos del feminismo institucionalista fueron identificados como parte de las corrientes políticas críticas y uno como parte de las posmodernas/deconstructivistas. En el caso del feminismo de la igualdad este número desciende a cero.
} 
De hecho, el 73,7\% de los artículos considerados dentro del feminismo institucionalista son políticamente liberales, y $22 \%$ radicales. En el caso del feminismo de la igualdad $64,6 \%$ se inscriben dentro de la tendencia liberal y $35,4 \%$ en la tendencia radical. Por lo tanto, se observa una preminencia importante de procesos de teorización adscriptos a discursos liberales no problematizadores del sujeto que suelen atenerse, en su mayoría, al análisis del sesgo sexista en el funcionamiento de las instituciones y los dispositivos o herramientas para una tendencia hacia la igualdad dentro del orden sistémico.

No es menor considerar esto, si, siguiendo a Foucault $(1978,1979)$, el liberalismo es un tipo de racionalidad que se inaugura con la episteme moderna occidental y es el tropo propio de la biopolítica. Entonces, podemos inferir que parte importante de la producción feminista liberal (institucionalista y de la igualdad) se encierra en las condiciones de posibilidad del discurso moderno occidental.

Ahora bien, si $42,4 \%$ de $68 \%$ se concentran en tres registros, significa que sólo queda un $25,6 \%$ restante para los otros tipos de feminismos.

\section{Los intersticios de los feminismos de la tercera ola}

La tercera ola del feminismo se inaugura a partir de la década del '90 cuando los discursos de un feminismo unificado y homogéneo, disputado ya desde la praxis 426 política fundamentalmente por las feministas negras, comienza a ser teorizado de manera sistemática, traduciéndose esto en una imbricación entre teoría y práctica. A partir de esta década lx sujetx del feminismo se desagrega en múltiples identidades e, incluso, se plantea su crisis, y se critica su necesidad y sostenimiento de la mano de las teorizaciones deconstructivistas y postestructuralistas que discuten las narrativas universalizadoras y universalizantes.

Del crisol diverso que surge, como bien aclara Dietz, Muchas teóricas feministas arman combinaciones ingeniosas de estos discursos y métodos y crean sus propias posturas híbridas críticas e interpretativas; pero sería un error suponer (y nada aconsejable desear) que sus diversas posturas se reúnen en última instancia y de manera coherente bajo el adjetivo "feminista" como una categoría unitaria (2003, p. 180). 
Con la excepción del lesbofeminismo y el ecofeminismo ${ }^{26}$, cuya génesis se encuentra en la segunda ola, gran parte de los registros dispuestos en la variables "corrientes inscriptas dentro del lineamiento político" surgen durante la tercera ola y son, precisamente, las que no se corresponden con los feminismos antes mencionados.

Los artículos clasificados dentro de los feminismos decoloniales; teoría queer; feminismos indígenas; masculinidades; ecofeminismo; feminismos populares; feminismos negros; transfeminismos; y lesbofeminismo suman 116 artículos. Si a estos los cruzamos con la variable "lineamiento político predominante", obtenemos un total de 104 artículos distribuidos entre las corrientes críticas y la corriente posmoderna/deconstructivista, es decir el $89,6 \%{ }^{27}$.

Vemos entonces que existe una coherencia político-teórica en tanto los encuentros entre los diferentes tipos de corrientes y subcorrientes políticas poseen una congruencia ideológica y conceptual que permite su encuentro. Más aún cuando, si desagregamos esos 104 artículos, observamos que 80 de ellos se ubican en la corriente crítica y 24 en el deconstructivismo.

Esto se debe a que los feminismos decoloniales, indígenas, los estudios de las masculinidades, el ecofeminismo, los feminismos populares, negros, transfeministas y lesbofeministas no suelen desprenderse del sujeto y la situacionalidad en su teorización sino que, a grandes rasgos, desafían la idea de la sujeta mujer blanca, occidental, 427 burguesa y heterocisexual para plantear sus fisuras tras el rescate de otras formas de vida, existencias y subjetividades; siendo estas indígenas, villeras o populares, negras, trans, lesbianas, no-occidentales y masculinidades subalternizadas.

En cambio, el feminismo deconstructivista "quiebra y desmantela todas las categorías multívocas y preconstituidas (raza, color, clase, género) y 'los etcéteras' (Butler 1990: 143) que las teorías de la diversidad promueven como de importancia capital tanto cultural como políticamente" (Dietz, 2006, p. 195) por eso, la mayoría de los artículos de la teoría queer (21/25) se ubican en este último. Recordemos que la teoría queer supuso una crítica radical de las concepciones, prácticas y agendas del

\footnotetext{
${ }^{26}$ Ambos tienen la particularidad fundamental de que se acoplan a la producción teórica y el giro discursivo que plantea la tercera ola. Por lo que el ecofeminismo que en primer lugar nace de la mano del feminismo de la diferencia, tendrá un viraje hacia posturas no esencialistas; por su parte, el lesbofeminismo, de la mano de autoras como Adrianne Rich en su texto de 1980 "Heterosexualidad obligatoria y existencia lesbiana" y la francesa materialista Monique Wittig quien adopta conceptos del posestructuralismo lo cual puede verse en El pensamiento heterosexual y otros ensayos (1992)

27 De los 12 restantes, la mitad son parte del feminismo marxista, cinco del feminismo radical y uno del feminismo liberal.
} 
feminismo de la segunda ola y se propuso a desbaratar las estructuras e identidades hasta el momento sostenidas por los feminismos. A través de instrumentos como la deconstrucción y la genealogía cuestionan los dispositivos de normalización de la subjetividad con principal hincapié en los prlocesos de normativización de la sexualidad para mover y romper aquello que haya cristalizado.

Ahora bien, si este cruce lo complejizamos intersectando la variable epistemológica veremos que también se sigue una congruencia conceptual, de manera que todos los artículos inscriptos en los feminismos de la tercera ola, al constituirse como críticos de los discursos positivistas, dejan atrás al empirismo feminista en lo que a la producción del conocimiento refiere. Si tomamos los 104 artículos que surgen del cruce de estos con el deconstructivismo y los feminismos críticos, el $77 \%$ se inscriben en el punto de vista feminista y el 23\% en la epistemología posmoderna (de los cuales el 83\% es de teoría queer).

A contramano de esto, si observamos al feminismo institucionalista, de la igualdad y de la diferencia, ocurre exactamente lo contrario, la epistemología posmoderna no aparece en sus registros, pero sí el punto de vista feminista y el empirismo feminista. De hecho, de los 192 artículos que suman los artículos de estos tres tipos de feminismo, 139 son de la epistemología del punto de vista y 45 del empirismo feminista ${ }^{28 .}$

Ahora bien, tras dejar claro que el $42,4 \%$ de los artículos se nuclean en torno a tres grandes registros (con la pertinente aclaración de que el feminismo de la diferencia sólo se lleva el 2,9), debemos destacar ciertos números que llaman la atención. Los discursos heterodoxos que mayor raigambre en las RI con perspectiva de género tienen son los feminismos decoloniales y la teoría queer, no obstante, estos son el 8,6\% y 5,5\% de los artículos respectivamente.

El número disminuye aún más cuando nos trasladamos al resto de los feminismos que no superan el $3 \%$ de la totalidad de los artículos, a saber: feminismo indígena 2,6\%; masculinidades 2,4\%; ecofeminismo $2 \%$; feminismos populares $1,8 \%$, feminismos negros $1,3 \%$; transfeminismos $1,1 \%$; lesbofeminismo $0,2 \%$. Dicho de otra manera, siete perspectivas políticas diferentes ocupan menos del 12\% de los artículos o 52 artículos de 453.

\footnotetext{
${ }^{28}$ Los ocho restantes no se identifican.
} 


\section{Conjeturas finales}

Las preguntas que dieron origen a este trabajo de investigación versaron sobre la posibilidad (o no) de hablar de una (o varias) comunidad(es) epistémica(s) con perspectivas de géneros y feminismos latinoamericanos en el campo de las RRII, constituida con reivindicaciones y conceptualizaciones propias.

Debido a que los resultados arrojados en este trabajo correspondan a una primera etapa de investigación, la cual es posible complejizar en niveles posteriores cruzando más variables entre ellas por un lado; e introduciéndonos en los escritos relevados, por el otro; podríamos decir que aún nos encontramos en una etapa de interpretación, de conversaciones, dicho, en otros términos, en una etapa de hermenéutica, en donde podemos observar que prevalece la adscripción conceptual, teórica y política a los discursos ortodoxos de las ciencias del Norte Global, pero con algunos intentos de resignificaciones basadas en las experiencias regionales, y con un mínimo margen de conceptualización propia.

Partimos del supuesto de que el conocimiento se encuentra vinculado con las relaciones sociales y prácticas de las comunidades, desde los contextos y actividades en y a través de los cuales se desarrollan las ontologías, se adoptan estándares de evidencia y metodologías, se construyen teorías y se abandonan o excluyen otras. En este sentido, observamos que existe una comunidad epistémica iberoamericana en ciernes, con 429 trabajos de publicación principalmente en Brasil, Argentina, México y España, muy poco diversa en términos étnicos y de identidad de género, concentrada mayoritariamente en los ámbitos académicos de posgrado, especialmente en los campos de las RI y la Ciencia Política.

Respecto al debate sobre la mímesis/hibridación de producción autóctona de conceptos y formas de análisis en las RI, podríamos decir que nos encontramos en una etapa de escasos esfuerzos contrahegemónicos. Gran parte de las publicaciones tiene un tenor liberal, emmarcadas principalmente en los feminismos institucionalistas y de la igualdad. Esto nos indica un a priori en nuestra forma de ver y entender, y de cómo se producen los discursos feministas en las RI iberoamericanas.

A su vez, la mayor parte de los artículos reproducen al punto de vista como la posición epistemológica desde donde analizar los fenómenos que abordan. Lo alentador en la adopción de este enfoque es que, aunque nacido y teorizado en el norte, reivindica el conocimiento situado, por lo que su apropiación política e ideológica por parte de movimientos del Sur Global puede ser terreno fértil para la performatividad localizada en nuestras cartografías. 
En ese sentido, el hecho de que los artículos considerados dentro del empirismo feminista que reproduce a nivel epistemológico las formas de producción positivista y ortodoxa de la ciencia sólo ocupen el $11 \%$ del total puede implicar que, al menos, las posiciones acríticas de la ciencia son las menores.

Por otro lado, yendo a las subcorrientes políticas, con la excepción del feminismo indígena, el feminismo decolonial y el feminismo popular/comunitario, el resto tienen su génesis localizada en el Norte Global. No obstante, las mismas en algún punto fueron importadas a nuestras latitudes lográndose instancias de resignificación tanto prácticas como teóricas ${ }^{29}$.

Observando que buena parte de nuestro corpus teórico se enmarca las corrientes políticas liberales y radicales, nos indica que buena parte del mismo no es contestatario respecto al Norte Global, en tanto las premisas teóricas del liberalismo se enmarcan dentro de la modernidad, y el posicionamiento radical sólo observa los dispositivos sistémicos de dominación del patriarcado sobre la mujer como sujeta unidimensional.

$\mathrm{Si}$, entonces, consideramos que las corrientes críticas y deconstructivistas abordadas epistemológicamente desde el punto de vista o el posmodernismo son el terreno más fértil para dar margen a la proactividad situada en fenómenos del Sur entonces, como vimos, el número es más reducido y más aún lo sea, seguramente, una vez que se indague en lo escrito en ellos.

A partir del recorrido hecho hasta aquí, como una primera aproximación podríamos decir que las perspectivas y trayectorias sobre perspectiva de género(s) y feminismos iberoamericanos en RI se encuentran en un proceso de hibridación con tendencia hacia el centro en cuanto a la adopción y mimetización de los supuestos hegemónicos. Sin embargo, existen esfuerzos prometedores para poder subvertir este canon.

\footnotetext{
${ }^{29}$ Desde el lesbofeminismo con autoras como val flores, Virginia Cano, Margarita Pisano, Yuderkys Espinosa Miñoso, Ochy Curiel, Norma Mogrovejo, etc. Desde los transfeminismos con Mauro Cabral, Lohana Berkins, etc; el feminismo negro y chicano de Cherrie Moraga y Gloria Anzaldúa; Yayo Herrero, Lolita Chávez, Karina Bidaseca y Laura Zúñiga desde el ecofeminismo de las periferias; la teoría Cuir, queer y Cu(y)r de Diego Falconi, Yayo Herrero, Lolita Chávez, Karina Bidaseca y Laura Zúñiga y val flores nuevamente. Los estudios de las masculinidades son los únicos que no cuentan con un proceso de reapropiación teórico (y su reapropiación en términos de praxis es parcial) sistematizado en el Sur Global aunque podemos nombrar a autores como Luciano Fabri y Enrique Stola que en Argentina desde hace tiempo vienen impulsando el pensamiento de las masculinidades desde discursos del Sur.
} 


\section{Referencias bibliográficas}

Acharya, A. y Buzan, B. (eds.) (2010) Non-Western International Relations Theory. Perspectives on and beyond Asia, Abingdon, Routledge.

Acharya, A. (2013) "Dialogue and Discovery: In Search of International Relations Theories Beyond the West", en Millennium: Journal of International Studies, $\mathrm{n}^{\circ}$ 39(3), pp. 619-637.

Acheraïou, A. (2011) Questioning Hybridity, Postcolonialism and Globalization, Houndmills, Basingstoke, Palgrave Macmillan.

Ackermann, A. (2012) "Cultural Hybridity: Between Metaphor and Empiricism", en Wolfgang Stockhammer, Philipp (ed.) Conceptualizing Cultural Hybridization. A Transdisciplinary Approach, Berlin, Heidelberg, Springer-Verlag, pp. 5-25.

Addelson, K. P., y Potter E. (1991) “Making Knowledge." en Messer-Davidow, Ellen y Joan, Hartmann (ed.) Engendering Knowledge: Feminists in Academe, Knoxville, Tenn, Univ. of Tenn. Press.

Bello Ramírez, A. (2018). "Hacia una trans-pedagogía: reflexiones educativas para incomodar, sanar y construir comunidad” en Debate feminista. Pp 104-128.

Bhabha, H. (1994) The location of culture, Londres, Routledge.

Bleier, R. (1984) Science and Gender: A Critique of Biology and Its Theories on Women. New York, Pergamon Press.

Briceño Ruiz, J., y Simonoff, A. (2017) "La Escuela de la Autonomía, América Latina y la teoría de las relaciones internacionales", en Estudios Internacionales, $\mathrm{n}^{\circ} 186$, pp. 39-89.

Code, L. (1991) What Can She Know? Feminist Theory and the Construction of Knowledge, Ithaca and London, Cornell University Press.

Crawford, R. y Jarvis, D. (2001) International Relations Still an American Social Science? Towards diversity in International Thought, Albany, State University of New York Press.

Del Moral Espín, L. (2012). "En transición. La epistemología y filosofía feminista de la ciencia ante los retos de un contexto de crisis multidimensional", en e-cadernos Ces, n8.Pp 51-80.

Dietz, M. J. (2003). "Las discusiones actuales de la teoría feminista" en Annual Review of Political Science, vol 6. Pp. 179-224.

Fernández Zubieta, A. (2009) "El constructivismo social en la ciencia y la tecnología: las consecuencias no previstas de la ambivalencia epistemológica", en ARBOR Ciencia, Pensamiento y Cultura, pp.689-703. 
Flax, J. (1986). Gender as a Social Problem: In and For Feminist Theory. American Studies/Amerika Studien, Journal of the German Association for American Studies.

Foucault, M. (2008). Nacimiento de la biopolitica. Fondo de cultura económico. Buenos Aires, Argentina.

Gamba, S. B. (Ed.) (2007) Diccionario de Estudios de Género y Feminismos. Editorial Biblos.

Hankinson N., Lynn. (1993) "Epistemoloogical communities", en Alcoff, Linda y Elizabeth, Potter (ed.) Feminist Epistemologies, New York, Routledge, pp.121-161.

Harding, S. (2004), "Introduction: Standpoint Theory as a Site of Political, Philosophic, and Scientific Debate", en Sandra Harding (org.), The Feminist Standpoint Theory Reader: Intellectual and Political Controversies. London: Routledge, pp $1-16$.

Harding, S. (1986) The Science Question in Feminism, Ithaca, Cornell University Press.

Hintikka S., y Hintikka M. (ed.) Discovering Reality. Kluwer Academic Publishers, pp. 225-244.

Holsti, K. (1985) The Dividing Discipline: Hegemony and Diversity in International Theory, Londres, Allen \& Unwin.

Jaggar, A. (1983) Feminist Politics and Human Nature. Totowa, Rowman \& Allenheld. 432

Knorr-Cetina, K. (1996) “¿Comunidades científicas o arenas transepistémicas de investigación? Una crítica de los modelos cuasi-económicos de la ciencia”, en Redes, no 7, pp. 129-160.

Knorr-Cetina, K. (2005) La fabricación del conocimiento. Un ensayo sobre el carácter constructivista y contextual de la ciencia, Buenos Aires, Editorial UNQ.

Latour, B., y Woolgar, S. (1995) La vida en el laboratorio. La construcción de los hechos científicos, Buenos Aires, Alianza.

Longino, H. (1990) Science as Social Knowledge: Values and Objectivity in Scientific Inquiry, Princeton, Princeton University Press.

Rovetto, F., Camusso M., Caudana, L., y Figueroa, N. (2017). "Feminismos y Ciencias Sociales: propuestas pedagógicas y aportes críticos para revisar programas de formación en el grado" en Revista de Educación, n¹2. Pp. 119-126.

Salomón, M. (2002). "La teoría de las Relaciones Internacionales en los albores del siglo XXI: diálogo, disidencia, aproximaciones" en Revisa Electrónica de Relaciones Internacionales, $\mathrm{n}^{\circ} 4$. Pp. 1-59 
Sarthou, N. F. (2013). "Perspectivas sociológicas del quehacer científico. Revisión bibliográfica y enfoques del comportamiento de los investigadores", en Nómadas. Critical Journal of Social and Juridical Sciences, V.38(2), pp.193-220.

Scheman, N. (1983) "Individualism and the Objects of Psychology." en Harding,

Smith, D. (1987) The Everyday World as Problematic: A Feminist Sociology, Boston, Northeastern University Press.

Smith, S. (2000) "The discipline of international relations: still an American social science?”, en British Journal of Politics and International Relations, n²(3), pp. 374402.

Wade, P. (2000) Raza y etnicidad en Latinoamérica, Quito, Editorial Aby-Yala.

Wæver, O. (1998) "The Sociology of a Not So International Discipline: American and European Developments in International Relations", en International Organization, $\mathrm{n}^{\circ}$ 52, (4), pp. 687-727.

Whitley, R. (2012) La organización intelectual y social de las ciencias, Bernal, Editorial UNQ. 